\title{
Actividad de la lactato deshidrogenasa en fluído crevicular gingival y saliva en fumadores con periodontitis crónica
}

\section{Lactate dehidrogenase activity in gingival crevicular fluid and saliva of smoker with chronic periodontitis}

\author{
LEYVA HUERTA E R* \\ ESQUIVEL CHIRINO C* \\ MARÍN GONZÁLEZ G** \\ NEBLINA NORIEGA $M * *$ \\ OLIVARES TAPIA $\mathrm{S} * *$
}

\begin{abstract}
Leyva Huerta E R, Esquivel Chirino C, Marín González G, Neblina Noriega M, Olivares Tapia S. Actividad de la lactato deshidrogenasa en fluido crevicular gingival y saliva en fumadores con periodontitis crónica. Av Periodon Implantol. 2000; 21, 1:21-26
\end{abstract}

\section{RESUMEN}

Existe un alto porcentaje de enfermedad periodontal atribuido al hábito del tabaco. La respuesta del organismo a esta enfermedad incluye la liberación de enzimas intracelulares relacionadas con la muerte y destrucción celular, como la Lactato Deshidrogenasa (LDH). Objetivo: Comparar los valores de LDH en fluido crevicular gingival (FCG) y saliva de pacientes fumadores y no fumadores con Periodontitis crónica (PC). Metodología: Participaron 15 pacientes hombres mayores de 30 años; 6 fumadores, 6 no fumadores con PC y 3 sujetos control. Se recolectaron muestras de saliva total y de FCG de bolsas periodontales de $=6 \mathrm{~mm}$. El volumen del FCG se determino pesando las puntas de papel antes y después del muestreo obteniendo los valores en gramos y convertidos a unidades de volumen (ml). La actividad de LDH se determino por medio de espectrofotometría. Los resultados se convirtieron a unidades de actividad enzimática expresados en $\mathrm{mM}$. El volumen de FCG fue de 0,78 $\mu \mathrm{L}$ en sujetos sanos, en no fumadores con PC 1.12 y en fumadores con PC 1,32. Los valores de LDH correspondieron a; saliva de sujetos sanos 0,845, en no fumadores con PC 1,325 y en fumadores con PC 1,7895 mM. En FCG la actividad fue 0,4568 en sujetos sanos, en no fumadores con PC 0,987 y en fumadores con PC 1,2546 mM. Conclusiones: Las técnicas utilizadas permitieron determinar diferencia entre los valores de la actividad de LDH en ambos fluidos en fumadores y no fumadores con PC.

PALABRAS CLAVE: Lactato Deshidrogenasa, fluido crevicular gingival, saliva, periodontitis crónica.

\section{SUMMARY}

The response of the body to periodontal disease includes the production of intra-cellular enzymes like Lactate Dehydrogenase (LDH) which is released by damaged periodontal cells. The LDH can be found in the gingival crevicular fluid (GCF) and saliva as a result of cellular death and damage. Objective: To compare LDH activity in GCF and saliva of smoker and non-smoker patients with Chronic Periodontitis. Methods: 15 male patients (30 to 50 years of age) participated in this study; 6 smokers with Chronic Periodontitis, 6 non-smokers with Chronic Periodontitis and 3 health subjects. Samples of stimulated total saliva as well as four samples of gingival crevicular fluid while probing periodontal pockets ( $=6 \mathrm{~mm}$ deep) with standarized \#30 endodontic paper points were obtained. The

* Laboratorio de Patología Clínica y Experimental.

** Departamento de Periodoncia e Imploantología.

División de Estudios de Posgrado e Investigación, Facultad de Odontología, Universidad Nacional Autónoma de México. 
enzymatic activity of LDH was recorded and was quantified by espectrophotometry. Results: GCF volume was $0.78 \mu \mathrm{L}$ in health subjects, $1.12 \mu \mathrm{L}$ non-smokers with Chronic Periodontitis and $1.32 \mu \mathrm{L}$ smokers with Chronic Periodontitis. LDH activity in saliva of smoker was $1.7895 \mathrm{mM}$. non-smoker patients with Chronic Periodontitis 1.325 and health subjects $0.845 \mathrm{mM}$. In FCG the activity was $0.4568 \mathrm{mM}$ of health subjects , non-smokers with Chronic Periodontitis 0.987 and smoker with Chronic Periodontitis was $1.2546 \mathrm{mM}$. The enzymatic activity of LDH in gingival crevicular fluid and saliva was greater in smoking patients compared to non-smoking patients. Conclusions: These results suggest that $\mathrm{LDH}$ activity in saliva and gingival crevicular fluid can be used to evaluate the progression of periodontal disease as well as the effects of smoking.

KEY WORDS: Lactate Dehidrogenase, gingival crevicular fluid, saliva, chronic periodontitis.

Fecha de recepción: 23 de junio 2008.

Fecha de aceptación: 1 de julio 2008.

\section{INTRODUCCIÓN}

Dentro de los factores de riesgo que inciden en el desarrollo y severidad de la enfermedad periodontal; se encuentra el tabaquismo asociado frecuentemente con el desarrollo de periodontopatías, las personas fumadoras tienen 2,7 veces más riesgo de desarrollar enfermedad periodontal que las no fumadoras (1-3). El tabaco puede alterar el equilibrio microbiológico bucal, incrementándose el número de bacterias anaerobias. Además por una serie de mecanismos irritativos, térmicos y químicos el tabaco lesiona las células de la mucosa bucal y ocasiona diferentes alteraciones; incluyendo mayores índices de placa, cálculo, así como gingivitis, periodontitis y alteración en la reparación y cicatrización de los tejidos. (4-7). Los fluidos corporales como la saliva y el Fluido Crevicular Gingival (FCG) están siendo utilizados para evaluar enfermedades sistémicas y realizar diagnósticos clínicos (8-10), así mismo existen estudios en los que se reporta que la saliva y el fluido crevicular gingival son de utilidad para realizar el diagnostico de la enfermedad periodontal debido a que se han identificado diversos marcadores $(2,3)$ que se podrían utilizar de manera rutinaria en la clínica para poder evaluar la progresión de esta enfermedad (1). Uno de los marcadores identificado es la Lactato Deshidrogenasa (LDH) enzima que normalmente se asocia al citoplasma de las células y sus valores se incrementan cuando existe daño en la membrana de las células durante la respuesta inflamatoria (4) . La LDH y el Aspartato aminotransferasa (AST) son enzimas cuya actividad esta correlacionada con la inflamación gingival y con la destrucción de los tejidos en la Enfermedad periodontal (11-15). Así mismo se ha estudiado la asociación entre los niveles de cotinina en saliva y fluido crevicular en pacientes fumadores y no fumadores con enfermedad periodontal (15) Los valores de cotinina en concentraciones 5 a 6 veces mas elevadas en fluido crevicular que en saliva explicaría la susceptibilidad a la destrucción de los tejidos periodontales en los fumadores (16). El objetivo de esta investigación fue determinar el volumen del FCG y la actividad de la LDH en saliva y FCG en un grupo de fumadores y no fumadores con Periodontitis Crónica.

\section{MATERIAL Y MÉTODOS}

\section{Población de estudio}

En este estudio fueron incluidos 15 sujetos del sexo masculino mayores de 30 años, 6 fumadores $>10$ cigarros por día con Periodontitis Crónica, 6 no fumadores con periodontitis crónica y 3 pacientes no fumadores sin enfermedad periodontal provenientes de la Clínica de Periodoncia de la División de Estudios de Postgrado e Investigación de la Facultad de Odontología de la UNAM. Se tomo como variable principal la Lactato Deshidrogenasa para detectar una diferencia de 0,4 $\mathrm{U} / \mathrm{ml}$ con una desviación estándar de $\pm 0,1$. La prueba fue para 2 muestras independientes de 2 colas con un alfa de 0,01 y un poder del $90 \%$.

Los pacientes participaron bajo consentimiento informado de acuerdo al Comité de Ética de la División de Estudios de Postgrado. Previo a la toma de las muestras se realizó historia clínica y examen periodontal a los pacientes participantes utilizando el Índice Gingival de Löe y Silness, se midió el sangrado al sondeo, la profundidad de bolsa y el nivel de inserción. Fueron 
excluidos del estudio pacientes bajo tratamiento médico o periodontal.

\section{Colección de las muestras de Saliva y FCG}

Previo a la toma de saliva se le pidió al paciente que masticara papel de parafina durante 5 minutos para inducir la estimulación de la saliva y se recolectaron $5 \mathrm{~mL}$ de saliva total estimulada en tubos de propileno de 50 $\mathrm{mL}$ (Costar USA), se centrifugaron a $1.000 \mathrm{~g}$ durante 10 minutos a $4^{\circ} \mathrm{C}$ para remover células escamosas y se recolecto el sobrenadante para realizar los experimentos de $\mathrm{LDH}$.

Las muestras de FCG se recolectaron de las zonas mesial, distal, vestibular, palatino o lingual de los estructuras dentarias en los pacientes sanos y de bolsas periodontales de $>4 \mathrm{~mm}$ con puntas de papel estériles estandarizadas de endodoncia del $\mathrm{N}^{\circ} 30$ (Hygienic USA) de acuerdo a la metodología descrita por N.J Medlicott, Paolantonio (6, 7). Se eliminaron los restos de placa dentobacteriana supragingival con cureta periodontal (HuFredy, USA) cuidando de no tocar la encía, se aisló la zona con rollos de algodón y se seco la superficie del diente con suave corriente de aire durante 5 segundos. Se insertaron las puntas $1 \mathrm{~mm}$ hacia el surco gingival durante 45 segundos sin ejercer excesiva presión, inmediatamente fueron transferidas a tubos eppendorf y guardados a $20^{\circ} \mathrm{C}$ hasta realizar los experimentos de $\mathrm{LDH}$, las puntas de papel que se contaminaron con placa dentobacteriana y sangre fueron descartadas de los experimentos.

El volumen del fluido crevicular se determino pesando las puntas de papel antes y después de la toma de la muestra en una balanza analítica (OHAUS USA), la diferencia de los valores obtenidos en gramos fue convertido hacia unidades de volumen $\mu \mathrm{L}$ considerando el valor de la densidad =1, la diferencia de peso mostró el volumen absorbido en las puntas de papel.

\section{Actividad de LDH}

Para determinar la actividad de $\mathrm{LDH}$ en la saliva se recolectaron $80 \mu \mathrm{l}$ del sobrenadante de saliva y se incubaron con 0,244 mM de nicotidamida adenín dinucleótido reducido $(\mathrm{NADH})$ en un amortiguador de Tris (81,3 $\mathrm{mM}$ ) y $\mathrm{NaCl}(203,3 \mathrm{mM})$ a un $\mathrm{pH} 8,6$. Las puntas de papel fueron incubadas en un sustrato con 0,244 mM de $\mathrm{NADH}$, se añadieron $170 \mu \mathrm{l}$ de piruvato $(9,76 \mathrm{mM})$ para las muestras de saliva y se les adicionó a las puntas de papel que contenían el fluido crevicular a $30^{\circ} \mathrm{C}$ en un volumen total de 1,0 m山, las muestras se leyeron en un espectofotómetro en un rango de absorbancia de 340 $\mathrm{nm}$ a intervalos de 15 segundos durante 2 minutos. En presencia de LDH el piruvato es reducido a lactato con la oxidación simultánea de $\mathrm{NADH}$, lo que represento el NADH consumido el cual es directamente proporcional a la actividad de LDH en la saliva y en las puntas de papel con las muestras. La técnica utilizada y las reacciones enzimáticas se muestras en el esquema 1.

Los resultados obtenidos en el espectofotómetro se convirtieron hacia unidades de actividad enzimática que corresponden a ( 1 unidad=umol de NAD+ que se incrementa por minuto a $30^{\circ} \mathrm{C}$ ) y son expresados como el total de la actividad de LDH (umol unidades/L) por muestra (12). Se obtuvieron los valores promedio la desviación estándar.

\section{RESULTADOS}

\section{Volumen de FCG}

Con el propósito de determinar el volumen y evaluar las diferencias del fluido crevicular gingival en las muestras obtenidas en las puntas de papel. Se obtuvo el promedio y la desviación estándar de los valores obtenidos expresados en $\mu \mathrm{L}$ de los 3 grupos. El volumen del fluido crevicular fue mayor en el grupo de

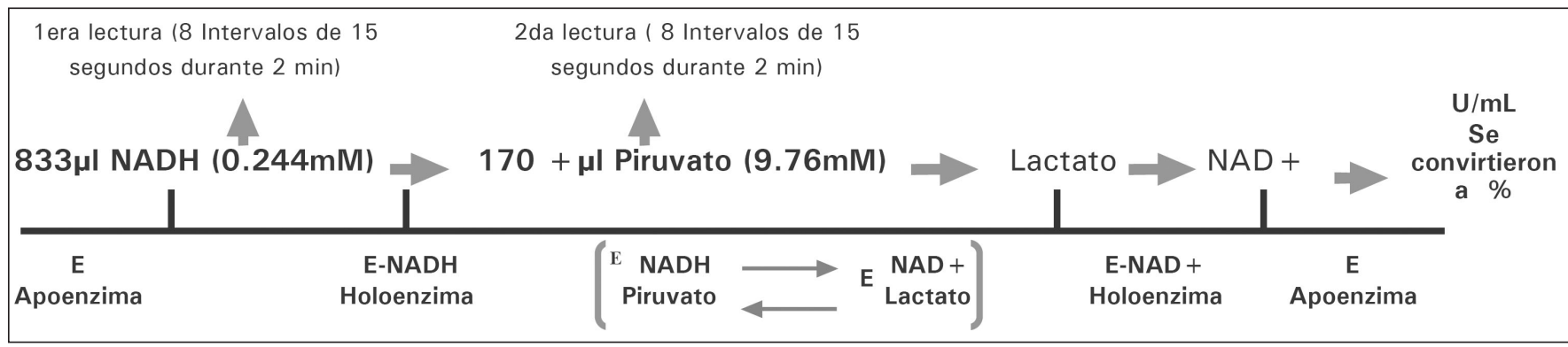

Esquema 1: Reacciones enzimáticas de la LDH.. (Obtenido de http://es.wikipedia.org/wiki/Lactato_deshidrogenasa) 
sujetos fumadores con Periodontitis Crónica que en los que sólo presentaban enfermedad periodontal. Se observó el incremento del volumen de fluido crevicular en el grupo de pacientes no fumadores con enfermedad comparado con el grupo control (Figura l).

\section{Actividad de LDH en el FCG}

Con el objetivo de evaluar la actividad de la LDH en el FCG se analizaron las puntas de papel con las muestras de los diferentes grupos de pacientes (Figura 2).

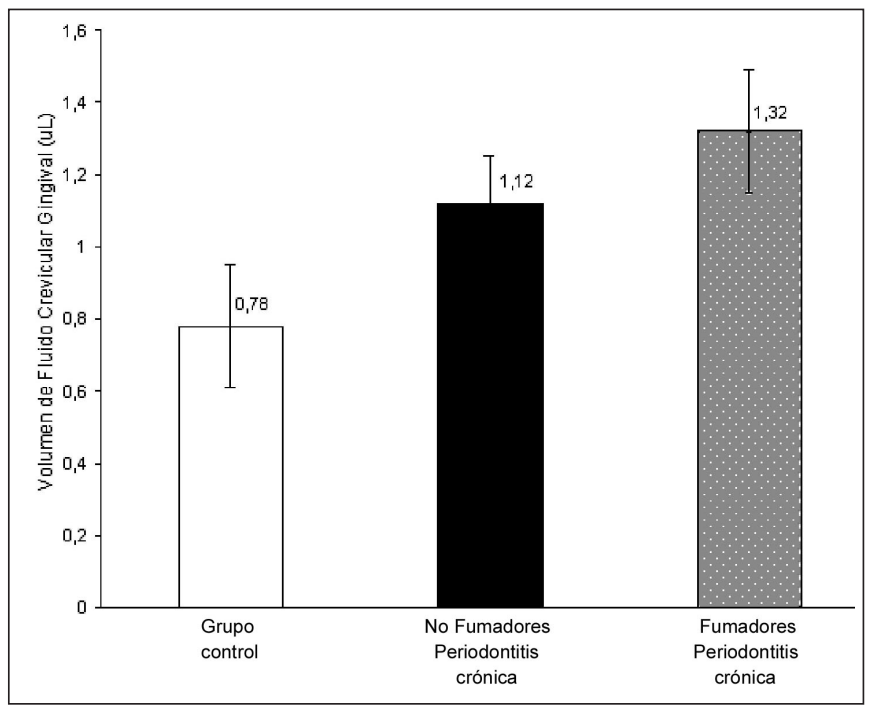

Fig. 1: Volumen de FCG expresado en $(\mu L)$ en los tres grupos de estudio.

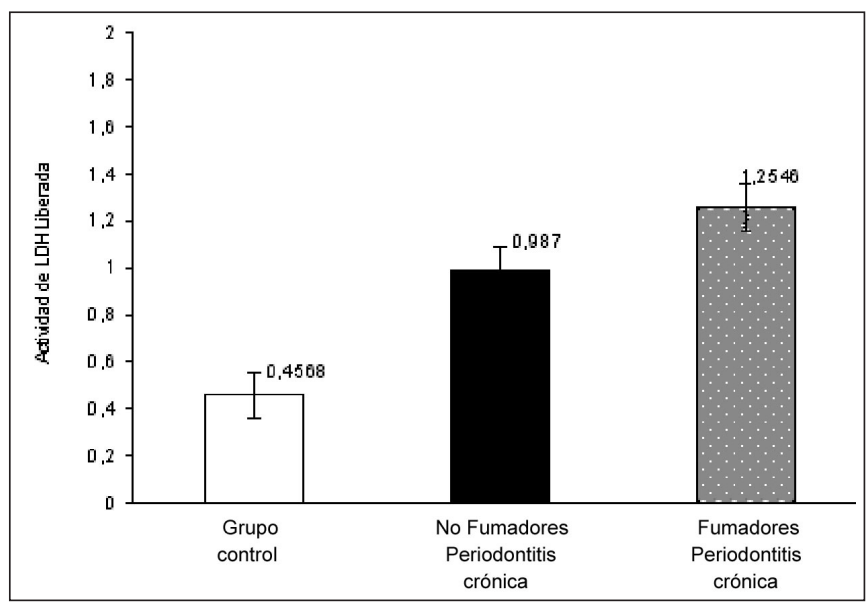

Fig. 2: Actividad de LDH en FCG en los grupos de estudio bajo diferentes condiciones.

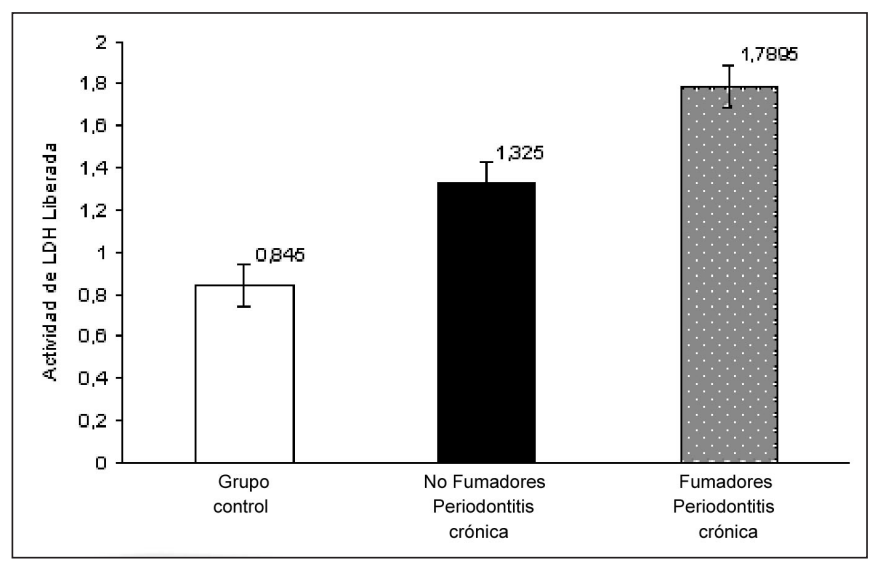

Fig. 3: Actividad de LDH liberada en la Saliva en los tres grupos de pacientes.

Se observó un incremento de la actividad de LDH liberada en los pacientes no fumadores con enfermedad periodontal con respecto al grupo control. En los pacientes con enfermedad periodontal fumadores existe un incremento de la actividad comparado con el grupo de no fumadores con enfermedad periodontal.

\section{Actividad de LDH en saliva}

En la saliva la actividad de la LDH liberada se observo mayor en los sujetos fumadores con Periodontitis crónica al compararlo con los otros dos grupos (Figura 3).

\section{DISCUSIÓN}

Los fluidos corporales han sido utilizados para evaluar enfermedades sistémicas y la enfermedad periodontal, algunos estudios han demostrado $(4,10)$ que el fluido crevicular gingival y la saliva contribuyen a realizar el diagnóstico de la enfermedad periodontal (13) en donde han comparado los niveles de lactato deshidrogenasa en saliva entre grupos de pacientes con bolsas periodontales, y en grupos de pacientes con sitios sanos, se reporto que la actividad de LDH es mas alta en los pacientes con bolsas periodontales profundas que en los sujetos sanos. Nishida y cols (16). Reportaron que la progresión de la enfermedad periodontal podría estar asociada con los niveles de LDH en el fluido crevicular gingival.

La obtención de las muestra del fluido crevicular gingival es un método que se realiza con puntas de papel absorbentes estandarizadas diseñadas para el siste- 
ma Periotron 8000, sin embargo la obtención del fluido crevicular se puede llevar a cabo por el método de obtención con puntas de papel estériles de endodoncia del $\mathrm{N}^{\circ} 30$ de acuerdo al método descrito por $N$. J. Medlicott, Paolantonio $(6,7)$. En nuestros resultados encontramos que los valores del fluido crevicular se incrementan en el grupo de no fumadores con enfermedad periodontal con respecto al grupo control y se observa un mayor incremento en el grupo de fumadores con enfermedad periodontal (Figura 1).

La obtención de las muestras de fluido crevicular gingival y la saliva es un método que permite detectar enzimas que están involucradas en la enfermedad periodontal como en el caso de LDH, aspartato aminotransferasa, fosfatasa alcalina, fosfatasa ácida, sin embargo Nomura y cols (1) reportaron que al evaluar la actividad de estas enzimas, LDH es la enzima que mayor incremento en su actividad presenta en la saliva de pacientes con enfermedad periodontal. En este estudio pudimos observar que la actividad liberada de LDH en el fluido crevicular gingival se incrementa en el grupo de no fumadores con enfermedad periodontal a diferencia del grupo control (Figura 2), esto coincide con diversos estudios que han evaluado esta actividad, en los cuales encontraron incrementos en la actividad de LDH en el FCG de pacientes con enfermedad periodontal (3), sin embargo la actividad de LDH liberada se incrementa en el grupo de fumadores con enfermedad periodontal. La actividad de LDH en saliva mostró resultados similares a los obtenidos en el fluido crevicular gingival, los valores del grupo de pacientes no fumadores con enfermedad periodontal se incrementa con respecto a los valores del grupo control, y la actividad de LDH se incrementa en el grupo de fumadores con enfermedad periodontal (Figura 3). Los incrementos en la actividad de LDH liberada en el fluido crevicular y saliva se deben en respuesta al daño tisular y de la membrana celular en las células que participan en la enfermedad periodontal.

El tabaquismo asociado a la enfermedad periodontal es considerado un factor de riesgo favoreciendo la progresión de la enfermedad periodontal $(14,17)$. Aunque no se conocen todos los mecanismos del tabaquismo que actúan sobre la enfermedad periodontal, se ha reportado que permite que se favorezca la destrucción de los tejidos de soporte.

\section{CONCLUSIONES}

Los resultados obtenidos muestran que los niveles de $\mathrm{LDH}$ variaron en los 3 grupos de estudio de manera similar tanto en el fluido crevicular como en saliva. La actividad de LDH fue mayor en ambos fluidos en los fumadores al compararlo con los no fumadores con y sin enfermedad periodontal.

\section{BIBLIOGRAFÍA}

1. Nomura Y, TamakiY, Tanaka T, Arakawa H, Tsurumoto A, Kirimura K, Sato T, Hanada N, Kamoi K. Screening of periodontitis with salivary enzyme tests.J Oral Sci. 2006 Dec;48(4):177-83.

2. Ligtenberg AJ, de Soet JJ, Veerman EC, Amerongen AV. Oral diseases: from detection to diagnostics. Ann N Y Acad Sci. 2007 Mar; 1098:200-3

3. Lamster IB, Ahlo JK. Analysis of gingival crevicular fluid as applied to the diagnosis of oral and systemic diseases. Ann NY Acad Sci. 2007 Mar;1098:216-29.

4. Nagler RM, Lischinsky S, Diamond E, Klein I, Reznick AZ. New insights into salivary lactate dehydrogenase of human subjects. J Lab Clin Med 2001;137:363-9.

5. Hatipoglu H,Yamalik N, Berberoglu A, Eratalay K. Impact of the distinct sampling area on volumetric features of gingival crevicular fluid. J Periodontol. 2007 Apr;78(4): 705-15.

6. N.J Medlicott, I.g. Tucker, M.j. Rathbone, D.W. Holborow. Determination of small sample volumes in the analysis of drugs at specific sites in oral cavity. J. Periodontal Res 1995;30:144-6.

7. Michele Paolantonio, Giancinto Di Placido, Vicenzo Tumini, Mauro Di Stilio, Alessandro Contento, Giuseppe Spotto. Aspartate Aminotransferase Activity in Crevicular Fluid From Dental Implants. J Periodontol 200;71:1151-7.

8. Miller CS, King CP Jr, Langub MC, Kryscio RJ, Thomas MV.Salivary biomarkers of existing periodontal disease: a cross-sectional study. J Am Dent Assoc. 2006 Mar;137 (3):322-9.

9. Taba M Jr, Kinney J, Kim AS, Giannobile WV. Diagnostic biomarkers for oral and periodontal diseases. Dent Clin North Am. 2005 Jul;49(3):551-71.

10. Zappacosta B, Manni A, Persichilli S, Boari A, Scribano D, Minucci A, Raffaelli L, Giardina B, De Sole P. Salivary thiols and enzyme markers of cell damage in periodon- 
tal disease. Clin Biochem. 2007 Jun; 40(9-10):66 1-5. Epub 2007 Jan 26.

11. Perinetti G, Serra E, Paolantonio M, Brue C, Meo SD, Filippi MR, Festa F, Spoto G. Lactate dehydrogenase activity in human gingival crevicular fluid during orthodontic treatment: a controlled, short-term longitudinal study. J Periodontol. 2005 Mar;76(3):41 1-7.

12. Alonso de la Peña V, Diz Dios P, Lojo Rocamonde S, Tojo Sierra R, Rodríguez-Segade S. A standardised protocol for the quantification of Lactate Deshydrogenase activity in saliva. Arch Oral Biol. 2004 Jan;49(1):23-7.

13. Gómez SC, Piccinin FB, Oppermann RV, Susin C, Nonnenmacher CI, Mutters R, Marcantonio RA. Periodontal status in smokers and never-smokers: clinical findings and real-time polymerase chain reaction quantification of putative periodontal pathogens. J Periodontol. 2006 Sep;77(9):1483-90.

14. Chen X, Wolff L, Aeppli D, Guo Z, Luan W, Baelum V, Fejeskov O. Cigarette smoking, salivary/gingival crevicular fluid cotinine and periodontal status. A 10-year longitudinal study. J Clin Periodontol. 2001 Apr;28 (4):331-9.

15. Hanioka T, Matsuse R, ShigemotoY, Ojima M, Shizukuishi S. Relationship between periodontal disease status and combination of biochemical assays of gingival crevicular fluid. J Periodontal Res. 2005 Aug;40(4):331-8.

16. Nishida N, Yamamoto Y, Tanaka M, Maeda K, Kataoka K, Nakayama K, Morimoto K, Shizukuishi S. Association between passive smoking and salivary markers related to periodontitis. J Clin Periodontol. 2006 Oct;33(10):71723. Epub 2006 Aug 3.

17. Nagler R, Lischinsky S, Diamond E, Drigues N, Klein I, Reznick AZ. Effect of cigarette smoke on salivary proteins and enzyme activities. Arch Biochem Biophys. 2000 Jul 15;379(2):229-34.

\section{CORRESPONDENCIA}

Elba Rosa Leyva Huerta

Laboratorio de Patología Clínica y Experimental

División de Estudios de Posgrado e Investigación

Facultad de Odontología

Universidad Nacional Autónoma de México

Circuito Institutos S/N Ciudad Universitaria.

Delegación Coyoacán

CP04519. México DF. MEXICO

Tel.56225560. FAX 55503497

docelbaleyva@hotmail.com 